\title{
Wenn Ihr nicht werdet wie die Kinder ...
}

\section{Regressive Phänomene in Religionen}

\section{Wilfried Ruff}

Online publiziert: 6 . November 2020

(C) Springer Medizin Verlag GmbH, ein Teil von Springer Nature 2020

Zusammenfassung Der Beitragstitel entstammt einem Zitat aus dem 18. Kapitel des Matthäus-Evangeliums und fordert zu einer Regression auf, die für das Christentum in seiner Geschichte gefährliche Folgen hatte. Am Beispiel des Christentums werden religionspsychologisch einige regressive Phänomene in ihren benignen und malignen Auswirkungen für Religionen diskutiert. Dafür nutzt der Autor das Modell des Bezogenseins von Daniel Stern.

\section{If you do not become like the children ...}

Regressive phenomena in religions

Abstract The title of this article is taken from a quotation from the 18th chapter of the Gospel of Matthew and calls for a regression which has had dangerous consequences for Christianity throughout history. Using Christianity as an example, some regressive phenomena are discussed in terms of their benign and malignant effects on religions. The author uses Daniel Stern's model of being related.

\section{Hermeneutische Überlegungen}

Der Vers aus dem Matthäus-Evangelium lautet vollständig: „Wenn ihr nicht umkehrt und werdet wie die Kinder, werdet ihr nicht in das Himmelreich hineinkommen“ (18,3). Dieses wahrscheinlich originale Jesuswort, dessen Übersetzung aus

Der Text war ursprünglich verfasst als Vortrag für den DPG-Kongress ,Zwischen Sehnsucht und Angst - Regressive Bewegungen im analytischen Feld“" in Marburg 2020, der wegen der COVID-19-Pandemie abgesagt werden musste.

Dr. med. et theol. W. Ruff $(\bowtie)$

Am Stadtpark 1, 91154 Roth, Deutschland

E-Mail: ruff@posteo.de 
dem Griechischen ins Deutsche nie Kontroversen ausgelöst hat, ist inhaltlich eine Provokation. Es fordert zu einer Umkehr auf. Umkehr auf welchem Weg? Umkehr im Denken, in der Gesinnung, im Verhalten, im Handeln? Radikal wie auch sonst oft die Evangelien formulieren, wird hier eine grundsätzliche Umkehr des Angesprochenen auf seinem individuellen Lebensweg gemeint sein. Ganz fremd dürfte das Psychoanalytikern nicht sein, wenn statt Umkehren das Wort Reflektieren (Zurückbiegen/Zurückwenden) gesetzt wird. Reflektieren - auch über Konflikte in der Kindheit - wird in Psychoanalysen von Therapeuten und Patienten erwartet. Aber doch nicht eine Rückwärtsbewegung in das Kindsein hinein und dann noch mit dem Versprechen einer Utopie in einer ungewissen Zukunft!

Mit diesem versprochenen Himmelreich wird meist ein Paradies ohne Leid und Not assoziiert. Denn das bedient drei Grundbedürfnisse, die uns aus dem frühkindlichen Erleben bekannt sind:

1. das Sicherheitsbedürfnis (Sandler),

2. das Bindungsbedürfnis (Bowlby),

3. das narzisstische Bedürfnis (Kohut).

Die Befriedigung dieser Bedürfnisse haben schon viele Ideologien versprochen (wie beispielsweise der Nationalsozialismus und der Kommunismus). Allerdings bezogen sich deren Versprechen auf eine irdische Wirklichkeit in einer Zeitspanne von Generationen. Dafür verlangten jene Ideologien harte Anstrengungen und das Schultern schwerer Lasten sowohl vom Einzelnen als auch vom Volk insgesamt.

Jesus forderte stattdessen bloß eine Rückwärtsbewegung wieder zum Kindsein, mit dem im Allgemeinen keine großen Leistungen und Anstrengungen verbunden werden - auch wenn wir heute wissen, was schon Kleinstkinder leisten, um sich Umwelt und Sprache anzueignen. Das war zu Zeiten Jesu nicht anders, vielleicht sogar noch mühsamer. Zwar galten Kinder in Israel nicht wie im griechisch-römischen Reich als bloße Sachen, über welche die Familie nach Belieben verfügen konnte. Kinder galten in Israel als Gaben und Segen Jahwes, die der Familie von ihm geschenkt wurden. Kinder wussten sich deswegen angenommen, ohne sich zuvor bewähren und behaupten zu müssen. Sie sollten ihren Eltern vertrauen, gehorchen und ihnen Ehrfurcht erweisen. Kinder galten als asexuell bis zu ihrem 9. Lebensjahr, auch noch im Christentum bis ins hohe Mittelalter (Hardt und Hoffmann 2006, S. 278). Deswegen sah man in ihnen das Ideal kultischer Reinheit verwirklicht (Lutterbach 2019).

Was mag Jesus in seiner Zeit mit seiner Forderung nach einer kindlichen Haltung gemeint haben, und was kann das heute für uns bedeuten? In der Exegese ist es die Frage nach dem ,Sitz im (jetzigen) Leben“. In unserer psychoanalytischen Arbeit gilt es, den Kontext zu beachten und die Technik der Deutung anzuwenden. Ich erinnere daran, dass Sigmund Freud diese Methode übernommen hat von der rabbinischtalmudischen Textauslegung ${ }^{1}$.

Im Kontext bei Matthäus hatten sich zuvor die Jünger darüber gestritten, wer von ihnen der Größte im Himmelreich sein werde. Darauf stellt Jesus als Antwort ein

\footnotetext{
1 Darüber hinaus organisierte Freud die Psychoanalyse wie eine Religion mit einem „Hang zum Dogmatismus und Konformismus“ (Pollak 2014, S. 1109).
} 
Kind - gleichsam als Modell - in ihre Mitte und kommentiert das mit den obigen Worten. Er meint tatsächlich Kinder seiner Zeit, die noch nicht wie seine Jünger miteinander konkurrieren und einander zu übertreffen suchen. Es geht Jesus also um ein kindliches Verhalten, das noch nicht um Leistung, Aufstieg und Gewinnermittlung kreist (Tatkraft, Leistung und Tüchtigkeit waren auch für Sigmund Freud die Ideale seines realistischen Weltbezugs). Die einzige Leistung, die er für wichtig erachtet, sieht er im Sich-Klein-Machen. Denn er fährt fort: „Wer sich so klein macht wie dieses Kind, der ist im Himmelreich der Größte“ (Mt 18,4). „Kleinmachen wie ein Kind" heißt aber nicht: wieder zu einem Kind werden. Es heißt eher, wieder die Sichtweise eines Kindes einnehmen, sodass ,umkehren“ einem Perspektivenwechsel entspräche. Entsprechend mahnte Paulus die Korinther ausdrücklich, als Erwachsene ihren Verstand zu gebrauchen, allerdings dabei ohne Bosheit zu sein wie Kinder (1 Kor 14, 20).

Bei Matthäus folgt dann eine erschreckende Drohung für Untaten an Kindern: „Wer eines von diesen Kleinen, die an mich glauben, verwirrt ${ }^{2}$, für den wäre es besser, wenn ihm ein Mühlstein um den Hals gehängt und er in der Tiefe des Meeres versenkt würde“ (Mt 18,6). Damit wird auf weitere Eigenschaften von Kindern hingewiesen: Sie sind noch sehr auf andere angewiesen, sie sind offen für Neues und oft sorglos, sie vertrauen noch leicht und sind naiv in ihren Erwartungen. Deswegen sind sie noch leicht zu verwirren und in die Irre zu führen, sind also in besonderer Weise auf Schutz angewiesen.

\section{Regression als Rückbewegung und Perspektivenwechsel}

Für Balint (1968) ist Regression ,not only an intrapsychic phenomenon, but also an interpersonal one“" (S. 146). In einer gutartigen Regression auf eine frühere Entwicklungsphase sieht er eine Art von Selbstheilungsversuch, durch den der Betreffende mit seinen Problemen ,erkannt“ werden wolle. Dagegen ziele eine bösartige Regression auf eine Triebbefriedigung durch einen Anderen. Balint entwickelte seine Überlegungen im Hinblick auf die Patienten-Therapeuten-Situation. Denn bis in die fünfziger Jahre des vorigen Jahrhunderts galt Regression als pathologischer Prozess. Erst Ernst Kris (1977) weitete den Regressionsbegriff aus: Regressionen im Dienste des Ichs geschähen auch zeitweise in schöpferischen Prozessen.

Balints Unterscheidung von Regressionen ließe sich auch auf normales Geschehen anwenden, wenn ,,benigne“ und „,maligne“ nicht als moralische Kategorien, sondern von ihren konstruktiven oder destruktiven Folgen her bedacht werden. Dabei folge ich dem dynamischen Entwicklungsmodell von Daniel Stern. Dieser beschrieb 1985 im eigenen Empfinden und dem gegenüber anderen verschiedene „Bereiche der Bezogenheit“, in denen Rückbewegungen immer wieder stattfinden können.

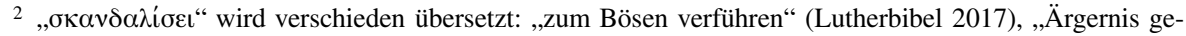
ben“ (Einheitsübersetzung), ,zu Fall bringen“ (Zürcherbibel) usw. Diese Tätigkeiten beginnen mit einer Verwirrung des Täters und führen zur Verwirrung des Opfers, weswegen ich das Wort „,verwirren“ gewählt habe.
} 
Aufgrund von Säuglingsbeobachtungen unterschied er fünf Erfahrungsbereiche im Selbsterleben und im Erleben seines intersubjektiven Bezogenseins:

4. den Bereich der Gefühle mit einem auftauchenden Selbstempfinden,

5. den Bereich der unmittelbaren Kontakte mit einem Kernselbstempfinden,

6. den Bereich der Gedanken mit einem subjektiven Selbstempfinden,

7. den Bereich der Worte mit einem verbalen Selbstempfinden und

8. den Bereich der Geschichten mit einem narrativen Selbstempfinden.

„In allen diesen Welten leben wir gleichzeitig. Sie überschneiden sich, verschwinden jedoch niemals ganz“ (Stern 1991 [1990], S. 19). Im Laufe des Lebens verändern sich diese Bereiche des Erlebens aufgrund von Erfahrungen und wirken untereinander und aufeinander ein. Dabei ,kann man eine Zeitlang nur dem einen Bereich, unter partieller Ausschließung der anderen, Beachtung schenken, aber auch in diesen anderen Bereichen findet Erleben weiterhin statt, unbemerkt, aber nicht unbemerkbar“ (Stern 1992 [1985], S. 53). Um einen Bereich besonders beachten zu können, muss das Erleben von den übrigen Bereichen abgezogen werden. Eine derartige Bewegung nenne ich einen Perspektivenwechsel, der entweder regressiv zu früher entstandenen Bereichen oder progressiv zu später entwickelten führen und sich benigne oder maligne auswirken kann (Ruff 2017). Weil sich alle Erfahrensbereiche im ganzen Leben weiterentwickeln, sind sie jederzeit für Dysfunktionen oder Deformationen anfällig (Stern 1992 [1985], S. 361).

1. Ein Beispiel für eine benigne Rückbewegung ist das kreative Schaffen. Denn ,jegliches Lernen und schöpferische Tun nimmt seinen Ausgang im Bereich der auftauchenden Bezogenheit" des Selbstempfindens und der Gefühle (Stern 1992 [1985], S. 103). Eine maligne Rückbewegung in diesen Bereich geschieht, wenn aufgrund hoher Triebspannung Gefühlsstürme zu einem inneren Chaos führen, und die Grenzen zwischen Innen und Außen verschwimmen.

2. Eine Rückbewegung in den Bereich der unmittelbaren Kontakte bewirkt ein Selbsterleben von intensiver Nähe zu einem Anderen im Hier und Jetzt mit Gefühlen von Geborgenheit und Trost, das bei zu starker Intensität und zu langer Dauer maligne in Selbstverlust umschlagen kann.

3. Gerät der Bereich in den Vordergrund, in dem die eigene Gedankenwelt von der Anderer unterschieden ist, erlebt der Betreffende (noch nonverbal) entweder großes Vertrauen mit viel Empathie für den Anderen oder er zeigt ein ausgeprägtes Misstrauen mit dem Bedürfnis, den Anderen zu beherrschen.

4. Beim Vorherrschen des verbalen Bereichs steht ein riesiger Freiraum an kommunikativen Möglichkeiten des Verstehens, Deutens und Sich-Abgrenzens (verbunden mit symbolischem Denken) zur Verfügung. Er ist aber auch prädestiniert für Missverständnisse und Konflikte, für Trennungs- und Verlassenheitsgefühle bis zum Verlust des Ganzheitserlebens.

5. Ist die Aufmerksamkeit vorwiegend auf den fünften Bereich der Geschichten gerichtet, geht es um die eigene Identitätsbildung im Narrativen. Erinnerungen und Wirklichkeitserleben werden im inneren Dialog bewertet, mit Fantasien und Träumen angereichert oder umgebildet. Dabei ist mystisches Erleben bis zur Selbst- 
entäußerung ebenso möglich wie eine traumatische oder psychotische Destruktion des narrativen Selbsterlebens.

\section{Folgen missbrauchter Macht}

Auf diesem Hintergrund können unterlassene Perspektivenwechsel regressive Phänomene in Religionen bewirken. Ich knüpfe zunächst an Erlebnisse in der katholischen Kirche aus meiner Kindheit und Jugend an. Damals predigten die Pfarrer nur zu gerne über die Matthäusstelle 18,3 als vorbildliches Verhalten für das Gottesvolk in den Kirchbänken. So wurden auch meine Eltern und alle erwachsenen Gläubigen zu Bescheidenheit, Demut und Schicksalsergebenheit, zu moralischem und religiösem Gehorsam, wie es sich für Kinder zieme, ermahnt. Widerrede gab es nicht. Für jene Tugenden, die alle in Abhängigkeit von der „Kirche“ halten sollten, wurde das Himmelreich, ein Land voll Milch und Honig, versprochen. Obwohl das in der kargen Nachkriegszeit erstrebenswert schien, war das für uns Heranwachsende meist keine Option: Wir wollten eher frei von der Bestimmung durch unsere Eltern werden. Doch sich gegen die kirchlichen Forderungen aufzulehnen, hieße: verdammt zu werden in die Hölle als dem Gegenstück zum Himmelreich. Und die Verdammnis wurde vom Prediger barock ausgemalt, um Angst und Furcht zu schüren. Der christliche Glaube war seit dem Mittelalter zum moralischen Damoklesschwert umgeschmiedet worden, zur Lebensversicherung gegen die Hölle. Das Gottesbild war mit seiner Überbetonung von Moral und Gehorsam zum Zerrbild eines unbarmherzigen Richters verkommen. Das wirkte noch bis in die Zeit nach dem 2. Weltkrieg - eine „Gottesvergiftung“, die Tilman Moser (1976, 2003) in seinen Büchern beschrieben hat. Danach distanzierten wir uns als Jugendliche und lästerten über die Betschwestern mit ihrer ,buckeligen Demut“, nicht ahnend, dass es sich dabei vielleicht um eine maligne Regression (nach Balint) handeln könnte.

Wenn gläubige Menschen sich derart ,demütig mit der geringfügigen Rolle ... bescheiden“ (Freud 1927, S. 355), nannte Sigmund Freud sie sogar „,irreligiös“. Denn eine solche Haltung entwickle sich aus Furcht aufgrund von Drohungen und Einschüchterungen (ebd., S. 370). Jene Fehlformen von buckeliger Demut führte Freud auf die Religion zurück, wobei er einem Glaubenden dennoch ,,bestimmte zärtliche Bindungen an die Inhalte der Religion“ (ebd.) zugestand.

Wenn wir diese Differenzierung Freuds konsequent weiterdenken, dann kann die Ursache für jene Fehlentwicklungen nicht die Religion als solche sein. Eine befriedigende Definition für die Religion als solche oder eine für alle Religionen umfassende Beschreibung haben die Religionswissenschaften bis heute nicht finden können. Dennoch: Wie verstehe ich Religion als solche? Ich schreibe ihr vier Wesenseigenschaften zu:

1. Sie ist eine Gemeinschaft, die im Glauben an ein die Wirklichkeit transzendierendes und ambivalent erfahrbares Heiliges Antworten sucht auf existenzielle Fragen von Menschen.

2. Sie verwirklicht sich im Kult mit verschiedenen Riten.

3. Sie gibt sich Regeln, die auf ethischen Werten beruhen (Wertekanon). 
4. Sie ist ein von Menschen geschaffenes Sozialgebilde, in dem sich institutionelle Elemente geschichtlich entwickeln. Damit ist Religion stets in Gefahr, dass sie in ihren konkreten Ausprägungen von Einzelnen oder Gruppen beherrscht und missbraucht wird.

So konnten sich auch in der Institution der katholischen Kirche menschenverachtende Machtstrukturen entwickeln und verfestigen. Ursprünglich gehörten alle - Kleriker wie Laien - in gleicher Weise zum Gottesvolk. Das von Jesus verkündete Kindschaftsverhältnis aller Gläubigen galt nur gegenüber Gott als dem Vater aller. Parallel zur geschichtlich bedingten Abgrenzung der Kirche von der weltlichen Herrschaft um die erste Jahrtausendwende begann der Klerus - in Stellvertretung für Gott - die Vaterrolle zu beanspruchen. Die kirchlichen Leitungsstrukturen mit entsprechenden Entscheidungsfunktionen gingen auf den Klerikerstand über, wobei nur die Orden eigenständig blieben. Das Kirchenvolk der Laien wurde weitgehend zu unmündigen Kindern, die von Klerikern geführt, erzogen und ermahnt werden mussten. Der Superioritätsanspruch der Kleriker führte zu ihrer rechtlichen und gesellschaftlichen Privilegierung. Ihre wohlorganisierten, hierarchischen Strukturen apersonal und legalistisch wie sie sich entwickelten (Klerikalismus) - ermöglichten das persönliche Verhalten der Amtsträger zu entpersonalisieren und diese in ihrem Tun von ihrer Verantwortung zu entlasten.

Die jahrhundertelange Entmachtung der Laien - auch in Bezug auf ihren Glauben ohne Rücksicht auf ihre Gewissensfreiheit - löste regressive Bewegungen im Kirchenvolk aus und erleichterte die Machtausübung durch die kirchlichen Institutionen. Damit einher ging eine zunehmende Idealisierung des Klerikerstandes, die ihn vor Angriffen schützen sollte: Er reklamierte für sich eine besondere Gotteskindschaft, weil er das Ideal der kultischen Reinheit verwirkliche (Lutterbach 2019). Als Martin Luther die hierarchisch gegliederte institutionalisierte Kirche aufgrund ihres rigiden Machtgebrauchs in Frage stellte, geriet die Amtskirche in Bedrängnis. Das wiederholte sich in den letzten drei Jahrhunderten durch Aufklärung, Säkularisation, Kulturkampf und Verfolgungen. ${ }^{3}$ Unter diesen Stressbedingungen zentralisierte sich die kirchliche Organisation immer mehr und zog sich wie in eine Wagenburg zurück. Probleme innerhalb der Kirche wurden ,ausschließlich mit dem eigenen methodischen Repertoire gelöst“", wozu heute allerdings auch eine Berufsgruppe wie die Psychoanalytiker neigt, wie es Pollak aufgezeigt hat (2014, S. 1119). Zwar spendeten die Kleriker dem Kirchenvolk weiterhin Orientierung, Geborgenheit und Trost, schränkten aber die Freiheit und Verantwortung des Einzelnen immer mehr ein. Zudem klafften Teile der offiziellen Lehre und die Realität mehr und mehr auseinander, was das 2. Vatikanische Konzil vor sechzig Jahren (1962-65) erkannte und verändern wollte. Wenn jedoch die Amtskirche in Verdacht geriet, Macht zu missbrauchen und Gewalt auszuüben, glaubte sie nicht den Opfern, sondern verdächtigte diese der Lüge. Die Wahrheitsfrage war zur Machtfrage geworden. Um ihr

\footnotetext{
3 Beispielsweise startete der Nationalsozialismus 1936-1937 mit rund 250 sogenannten Sittlichkeitsprozessen gegen Angehörige katholischer Orden und Weltpriester eine Kampagne zur Diskreditierung der Kirche. Sie fand ihren Höhepunkt in einer Rundfunkansprache Joseph Goebbels am 18.5.1937. Aufgrund dieser Kampagne sah sich die Kirche nach dem Krieg als Opfer der NS-Propaganda (Hockerts 2020).
} 
idealisiertes Selbstbild, das sie vor Ohnmacht, Scham und Schuld schützen sollte, nicht zu gefährden, wurde das Versagen von Klerikern verschleiert oder geleugnet. Sexuelle, destruktive und spirituelle Missetaten ${ }^{4}$ ihrer Mitglieder wurden mittels Projektion zurückgewiesen: Die Täter machten sich zu Opfern von Verleumdungen, Verführungskünsten und Nachstellungen vonseiten der Missbrauchten oder pathologisierten diese als infantil und hysterisch. Wir wissen heute, dass sich über $95 \%$ der Anzeigen Missbrauchter als begründet erwiesen haben (Rixen 2019, S. 46).

Zahlreiche erschreckende Missbrauchstaten wurden im letzten Jahrzehnt publik. Kirchliche Autoritätspersonen konnten die ihnen verliehene Macht und ihr Prestige ausnutzen, um Kinder und Jugendliche, aber auch erwachsene Ordensfrauen zu täuschen, zu betrügen und zu missbrauchen. Von 1946-2014 weist die MHGStudie Missbrauchstaten bei 3677 Minderjährigen nach, die von 4,4\% der Kleriker in Deutschland begangen wurden bei einer als hoch anzunehmenden Dunkelziffer (wikipedia.org/wiki/MHG-Studie). Anhand von Studienergebnissen wird vermutet, dass etwa ein Drittel aller Ordensfrauen sexuelle, körperliche oder geistliche Gewalt erlitten haben (Mathies 2019, S. 32).

Damit pervertierten die Täter ihren Glauben und christliche Tugenden (wie Gehorsam und Hingabe) zu kriminellen Waffen und missbrauchten auch ihre Religion. Sie verdrängten das Verdammungsurteil Jesu über diejenigen, die die Kleinen verwirren und zu Fall bringen.

Die Täter gerieten in ihrem Machtmissbrauch auf tiefere Bereiche ihres Bezogenseins, wenn sie ihre Opfer skrupellos verführten und nur noch als Mittel zur Befriedigung eigener Bedürfnisse missbrauchten. Wie Daniel Stern feststellte, können Gefühlsstürme bei Tätern aufgrund hoher Triebspannung zu einem inneren Chaos und zum Verschwimmen der Grenzen zwischen Innen und Außen führen, was einer malignen Zentrierung auf den ursprünglichen Bereich des auftauchenden Selbstempfindens entspricht. Erschreckend ist dabei, dass die meisten Täter kein Verantwortungsbewusstsein erkennen lassen und die Wiederholungsrate derartiger Missbräuche sehr hoch ist, was auch auf defiziente Entwicklungen in höheren Bereichen des Bezogenseins verweist. Gerechterweise muss ich darauf hinweisen, dass etwa $90 \%$ des Klerus nicht zu diesem Täterkreis gehören. Jedoch deckten manche von diesen aus falscher Loyalität die Täter. Sie fürchteten, die Kontrolle über ihre Macht, ihren Einfluss und ihre Sicherheit zu verlieren. Letztlich rührt diese Loyalität aber aus einem Gefühl und Bewusstsein, eine auserwählte, doch bedrohte Gruppierung zu sein. Die malignen Folgen des in ihr fehlenden Perspektivenwechsels über Jahrhunderte haben nicht nur Bedürfnisse zur Beherrschung Anderer und eine männerbündische Kultur wuchern lassen, sondern gefährden jetzt endlich auch ihre eigene Position.

Wende ich das Entwicklungsmodell von Daniel Stern auf die Opfer an, so wurden die meisten erst durch den Machtmissbrauch in den Bereichen ihres Bezogenseins auf sich selbst und auf andere traumatisiert. Weil diese Schädigung vermutlich auch

\footnotetext{
4 Spiritueller Missbrauch geschieht, wenn Amtsträger Abhängigkeiten und Bedürfnisse einer Person emotional ausnutzen und dies begründen vom religiösen Glauben her, den sie umdeuten und als seelisches Druckmittel benutzen für das Erreichen eigener Zwecke und Ziele. Vgl. Schulz HA (2019) Perfide Konstrukte. Herderkorrespondenz 10:36-38.
} 
verbunden ist mit einer Fixierung in zumindest einem Bereich, bedeutet das für die Betroffenen eine maligne Entwicklungsstagnation in Form einer Abhängigkeit, die ihnen einen Perspektivenwechsel erschwert und bis in den Selbstverlust führen kann.

\section{Perspektivenwechsel als Chance}

Angesichts dieses Machtmissbrauchs im Christentum wage ich kaum darauf hinzuweisen, dass alle Religionen mehr oder weniger institutionell verfasst und damit in Gefahr des Missbrauchs sind. Und wo sollen da noch Chancen für progressive Perspektivenwechsel zu finden sein? Kann Regression auch Voraussetzung für Progression ,und damit höchst förderlich für eine (...) Weiterentwicklung“ oder sogar einen „Neubeginn“ werden, wie Winkler (1992, S. 99) in Anlehnung an Balint meinte?

Um Antworten darauf zu finden, komme ich auf die Forderung Jesu zurück, umzukehren und wie die Kinder zu werden. Ist das nicht auch eine faszinierende Möglichkeit, wieder wie ein Kind seine Umwelt ohne Leistungsforderungen erkunden zu können: versorgt mit Nahrung, Kleidung und Unterkunft, lustvoll und ohne Angst - eine tiefe Sehnsucht, die wohl jeder kennt. Und „Sehnsucht sucht nach einem Objekt, das größer ist als man selbst“ (Will 2017, S. 245). Dass ein Kind auf vielfältige Weise auch gemein, neidisch und bösartig sein kann, wird dabei ausgeblendet. Diese negativ zu wertenden Persönlichkeitsanteile können sich mit dem Erwachsenwerden immer stärker auswirken, vor allem in Machtmissbrauch und in Gewalt. Dies war für Jesus der Anlass, sich besonders den Rechtlosen und Unterdrückten zuzuwenden, aber auch die Erwachsenen zur Umkehr aufzurufen und sie wieder daran zu erinnern, wie sie sich als Kinder häufig erlebt hatten: voller Zuversicht und Vertrauen darauf, dass sie in ihrer Abhängigkeit von ihrer Umwelt von einem Stärkeren gehalten (,contained“) werden.

Wollte Jesus damit unserer Sehnsucht einen Erinnerungsraum schaffen? Oder mehr noch uns auffordern: „eine angemessene Balance zwischen der Möglichkeit, regressiven Tendenzen zu widerstehen [einerseits], und einer gewissen Durchlässigkeit und Flexibilität des Ich“ andererseits zu suchen (Geissler 2001, S. 248)? Denn selbst wenn sich ein Erwachsener wie ein Kind erlebt und verhält, versteht er sich weiter als Erwachsener.

An einem Beispiel, wie sich progressive und regressive Tendenzen im religiösen Bereich zeigen können, möchte ich an einem Traum verdeutlichen - ohne Anspruch, ihn in all seinen Facetten zu deuten. Ihn erzählte mir eine 53-jährige verheiratete Bekannte (keine Analysandin), eine gläubige Katholikin, vor etwa einem Jahr. Gerade im Traum erleben wir vielfältige und oft abrupte Perspektivenwechsel mit unterschiedlichen Folgen.

Ich träumte, ich sei wieder auf Besuch in Kamerun bei einem befreundeten Priester, der dort als Missionar tätig war. Er hatte eine große Kathedrale erbaut. Der filigrane neugotische Glockenturm war noch nicht fertig. Bei der Einweihung der Kathedrale diente ich beim Gottesdienst in einem Bischofsgewand. Dabei rutschte mir die Mitra immer wieder ins Gesicht. Danach putzte ich die 
Kirche. Dann war ich auf der Missionsstation. Aber da waren nur Kinder auf hölzernen Dreirädern. Ich bat sie, mir eines zu überlassen, damit ich damit nach Hause fahren könne. Die ganze Zeit war ein Mann bei mir, aber ich weiß nicht, wer es war.

Die Frau träumt sich nach Afrika in ein Entwicklungsland, in dem sie schon mehrfach in einer Missionspfarrei gewesen war. Im Traum steht da eine neue Kathedrale, von der nur der fein durchbrochene Turm noch nicht ganz fertig ist. Sie ist bei der Kirchweihe dabei - als wichtigste Person. Denn sie ist bischöflich gekleidet: eine Frau als Bischöfin, in der katholischen Kirche bisher unmöglich, aber von vielen Frauen gefordert. Bis hier zeigt der Traum eine unglaubliche Progression, die mit dem lustvollen Erzählen der Träumerin korrespondiert. Dann ein Perspektivenwechsel, über den die Träumerin zögerlicher und eher bedrückt erzählt: Der Bischofshut ist für sie zu groß, er rutscht ihr immer wieder ins Gesicht, hindert sie am Sehen. Wieder ein Perspektivenwechsel: Sie putzt auf den Knien die Kirche zur Buße für ihre Überheblichkeit. Danach wieder ein Perspektivenwechsel: Sie ist unter Kindern wie ein Kind. Sie möchte mitspielen, auch ein Dreirad wie die anderen haben. Mit dem will sie zurück, nach Hause in den Alltag umkehren. Bemerkenswert ist, dass sie die ganze Traumzeit, während jedem Perspektivenwechsel einen Begleiter neben sich spürt, den sie nicht zu identifizieren vermag. Der Begleiter, der nicht ihr Mann gewesen sei, habe ihr Halt gegeben, sie nicht allein gelassen: ein Engel, ein Vatergott?

„Umzukehren“ wird schon in der hebräischen Bibel immer wieder verlangt, wenn Israel als das von Jahwe auserwählte Volk Irrwege eingeschlagen hatte. Seine Propheten forderten, Israel als Volk solle sich wie ein behütetes Kind zum liebenden Vater verhalten (Jes. 63,16; Jer. 31,9; Hos. 11,1). Darüber hinaus lehrte Jesus, dass die Glaubenden schon Kinder Gottes, seines Vaters, seien. Gleiches beglaubigte der Evangelist Johannes: „Allen aber, die ihn (Jesus) aufnahmen, gab er Macht, Kinder Gottes zu werden, (und) allen, die an seinen Namen glauben“ (Joh 1,12; vgl. Mt 6,9; Gal 3,26).

Wenn Christen also ohnehin schon Kinder Gottes sind (1 Joh 3,1f.) - hat sich damit die Forderung Jesu nach einer Umkehr erübrigt? Auch für den Christen gilt, dass er sich immer wieder vom Beziehungsangebot Gottes abwendet, gegen ein (falsch verstandenes) Kindsein vor Gott protestiert. Gerade darin zeigt sich der Mensch in seiner Freiheit und Selbstbestimmung als autonomes Wesen gegenüber Gott. Denn er kann sich entscheiden, entweder ohne Gott nach Gutdünken zu leben, oder er kann sich zum Perspektivenwechsel entscheiden: zum Kindsein beim Vatergott einlassen (Wilhelm v. Ockham, Erasmus). Doch es gibt noch ein Drittes, weil sich Negation und Affirmation nicht ausschließen: eine „,betweenness“, eine Beheimatung im „Zwischen“. In letzter Konsequenz kann Umkehr bedeuten: „Man findet Gott, wo man ihn loslässt." Was mit diesem Satz der spätmittelalterliche Theologe Meister Eckhart ausdrückte, könnte im Zen-Buddhismus dem absoluten „Nichts“ entsprechen, das jede verdinglichte religiöse Sprache ausschließt und in der Mystik als averbale Anschauung erfahren wird.

Perspektivenwechsel und Umkehr gilt schließlich ebenso für religiöse Institutionen gemäß dem aus der evangelischen Tradition entlehnten Leitsatz ,ecclesia semper 
reformanda", die Kirche muss ständig verändert werden. Das heißt nicht, sich bloß an neue gesellschaftliche Veränderungen anzupassen oder lediglich Strukturen zu verändern. Vielmehr bedeutet das für religiöse Institutionen, immer wieder im Vertrauen und in der Zuversicht ihrer transzendenten Verankerung über sich selbst offenkritisch zu reflektieren und sich von außen hinterfragen zu lassen. Damit dies geschehen kann, müssen Institutionen anscheinend ähnlich wie Individuen erst in eine Krise von innerer Not mit Angst und Beschämung geraten, um sich zu besinnen und nach einer Hilfe vonseiten Anderer Ausschau zu halten ${ }^{5}$. Aus der anschließenden Entgrenzung und einer Belebung des religiösen Bezogenseins kann dann Umkehr und Erfahrung von Rettung erfolgen, was Herbert Will (2017) als religiöse Transgression bezeichnet. Für Religionen mit ihren Strukturen ist Voraussetzung für ihre immer wieder notwendige Erneuerung eine Rückbesinnung auf ihre Ursprünge im Bewusstsein ihres Kleinseins vor dem Gott, der in ihren Heiligen Schriften bezeugt ist als Schöpfer und Erlöser (und der ist im Christentum Jesus Christus).

Interessenkonflikt W. Ruff gibt an, dass kein Interessenkonflikt besteht.

\section{Literatur}

Balint M (1968) The basic fault: therapeutic aspects of regression. Tavistock, London

Freud S (1927) Die Zukunft einer Illusion Bd. 14, S 325-380 (GW)

Geissler P (2001) Mythos Regression. Psychosozial, Gießen

Hardt J, Hoffmann SO (2006) Kindheit im Wandel - Teil I: Antike bis zur Neuzeit. Prax Kinderpsychol Kinderpsychiatr 55(4):271-279

Hockerts HG (2020) Sittlichkeitsprozesse gegen Ordensangehörige und Priester in der NS-Zeit, FAZ 242, 17.10.20, S. 8.

Kris E (1977) Die ästhetische Illusion. Phänomene der Kunst in der Sicht der Psychoanalyse. Suhrkamp, Frankfurt/M.

Lutterbach H (2019) Werdet wie die Kinder. Spiritualität und Missbrauch. Herderkorrespondenz 1:48-51

Mathies H (2019) Das Schweigen beenden. Herderkorrespondenz 12:31

Moser T (1976) Gottesvergiftung. Suhrkamp, Frankfurt/M.

Moser T (2003) Von der Gottesvergiftung zu einem erträglichen Gott. Psychoanalytische Überlegungen zur Religion. Kreuz, Stuttgart

Pollak T (2014) Psychoanalyse als Religion? Zur kirchlichen Verfasstheit psychoanalytischer Institutionen. Psyche 68(11):1108-1131

Rixen S (2019) Verantwortung übernehmen. Herderkorrespondenz 12:43-46

Ruff W (2017) Perspektivenwechsel in Psychoanalyse und Religion. Reflexionen über ethische Fragen und Weltanschauungen. Psychosozial, Gießen

Stern DN (1991) Tagebuch eines Babys. Was ein Kind sieht, spürt, fühlt und denkt. Piper, München

Stern DN (1992) Lebenserfahrung des Säuglings. Klett-Cotta, Stuttgart

Will H (2017) Religiöse Erfahrung als Transgression. Ein Gedicht Edith Jacobsons aus nationalsozialistischer Haft als Beispiel. Psyche 71(3):235-259

Winkler K (1992) Werden wie die Kinder? Christlicher Glaube und Regression. Mainz, Grünewald

Dr. med. et theol. Wilfried Ruff Arzt für Psychiatrie und psychotherapeutische Medizin. Dreiundzwanzig Jahre lang Ärztlicher Direktor der Klinik Wittgenstein. Er war lange Jahre Vorsitzender des von ihm 1987 gegründeten Ausbildungsinstituts für Psychoanalyse und Psychotherapie Siegen-Wittgenstein, in dem er auch Lehranalytiker und Dozent ist. Veröffentlichungen zur stationären Psychotherapie, zu ethischen Grundfragen sowie zum Verhältnis zwischen Religion und Psychoanalyse.

\footnotetext{
5 Papst Franziskus hat diesen Weg mit seinem Brief vom 20. August 2018 an das ganze christliche Volk beschritten (http://w2.vatican.va/content/francesco/de/letters/2018/documents/papa-francesco_20180820_ lettera-popolo-didio.html).
} 